\title{
Vessel wall magnetic resonance imaging findings and surgical treatment in nilotinib-associated cerebrovascular disease: A case report
}

\author{
KOHEI SUZUKI ${ }^{1}$, JUNKOH YAMAMOTO ${ }^{1}$, SHINGO KAKEDA ${ }^{2}$, SEISHIRO TAKAMATSU $^{1}$, RYO MIYAOKA ${ }^{1}$, \\ TAKEHIRO KITAGAWA $^{1}$, TAKESHI SAITO ${ }^{1}$, YOSHITERU NAKANO ${ }^{1}$ and SHIGERU NISHIZAWA ${ }^{1}$ \\ Departments of ${ }^{1}$ Neurosurgery and ${ }^{2}$ Radiology, University of Occupational and Environmental Health, \\ Kitakyushu, Fukuoka 807-8555, Japan
}

Received September 19, 2018; Accepted November 13, 2018

DOI: $10.3892 / \mathrm{mco} .2018 .1780$

\begin{abstract}
Nilotinib, a second-generation tyrosine kinase inhibitor, is considered as one of the most effective drugs for the treatment of chronic myeloid leukemia (CML); however, the use of nilotinib has been reported to be associated with vascular adverse events, such as peripheral arterial occlusive disease and ischemic heart disease. Moreover, there are few reports on cerebral vascular disease associated with nilotinib use. We herein describe the case of a 55-year-old male patient with CML, who presented with cerebral infarction and severe cerebrovascular stenosis that developed during nilotinib treatment. The patient was diagnosed with cerebral infarction and severe stenosis of the intracranial arteries associated with nilotinib use. Vessel wall magnetic resonance imaging (VW-MRI) revealed diffuse concentric thickening of the vessel wall, unlike ordinary patterns of atherosclerosis. The patient underwent direct revascularization (superficial temporal artery to middle cerebral artery bypass) and was successfully treated without recurrence. Based on this rare case, VW-MRI may be
\end{abstract}

Correspondence to: Dr Kohei Suzuki, Department of Neurosurgery, University of Occupational and Environmental Health, 1-1 Iseigaoka, Yahatanishi-ku, Kitakyushu, Fukuoka 807-8555, Japan

E-mail: s-kohei@clinc.uoeh.ac.jp

Abbreviations: CML, chronic myeloid leukemia; CVD, cerebral vascular disease; DDR1, discoidin domain receptor 1; ICA, internal carotid artery; LDL, low-density lipoprotein; MCA, middle cerebral artery; MMR, major molecular remission; MRA, magnetic resonance angiography; MRI, magnetic resonance imaging; PAOD, peripheral arterial occlusive disease; PDGF-R, platelet-derived growth factor receptor; STA, superficial temporal artery; SPECT, single photon-emission computed tomography; TKI, tyrosine kinase inhibitor; VAE, vascular adverse event; VW-MRI, vessel wall magnetic resonance imaging

Key words: cerebral vascular disease, nilotinib, cerebrovascular stenosis, vessel wall magnetic resonance imaging, surgical revascularization used to detect the morphological changes of the intracranial arteries that are associated with nilotinib use. Moreover, surgical revascularization may improve the prognosis of nilotinib-associated cerebrovascular diseases, such as severe stenosis or occlusion of the main trunk of the cerebral arteries, that cause brain ischemia.

\section{Introduction}

Chronic myeloid leukemia (CML) is caused by uncontrolled proliferation of hematopoietic cells of the myeloid lineage and the presence of the BCR/ABL1 fusion oncogene. Tyrosine kinase inhibitors (TKIs) block the binding of adenosine triphosphate to BCR/ABL1 tyrosine kinase, thus inhibiting its activity (1). Imatinib, a first-generation TKI, has been considered as the standard treatment for CML (2); however, some patients develop resistance to imatinib caused by point mutations of the BCL/ABL1 chromosome. Compared with imatinib, nilotinib, a second-generation TKI, is highly effective and relatively safe for patients with CML (3). Thus, nilotinib has been increasingly used as the gold standard therapy for imatinib-intolerant or -resistant CML and newly diagnosed chronic-phase CML (3). Recently, nilotinib-associated vascular adverse events (VAEs), such as peripheral arterial occlusive disease (PAOD), have been reported $(1,4,5)$. Prospective reports have described an increased rate of VAEs among patients treated with nilotinib compared with imatinib $(1,4)$, although cerebral vascular disease (CVD) associated with nilotinib use is extremely rare (6-10).

We herein describe a rare case of nilotinib-associated CVD in a patient with CML, and discuss the differential diagnosis and management of this condition.

\section{Case report}

A 55-year-old Japanese male patient who had been receiving nilotinib treatment for CML (300 mg twice per day for 3 years) developed transient left-sided weakness of the extremities 2 days prior to presentation (Fig. 1). The symptoms worsened, and the patient was admitted in January 2017 to the University of 


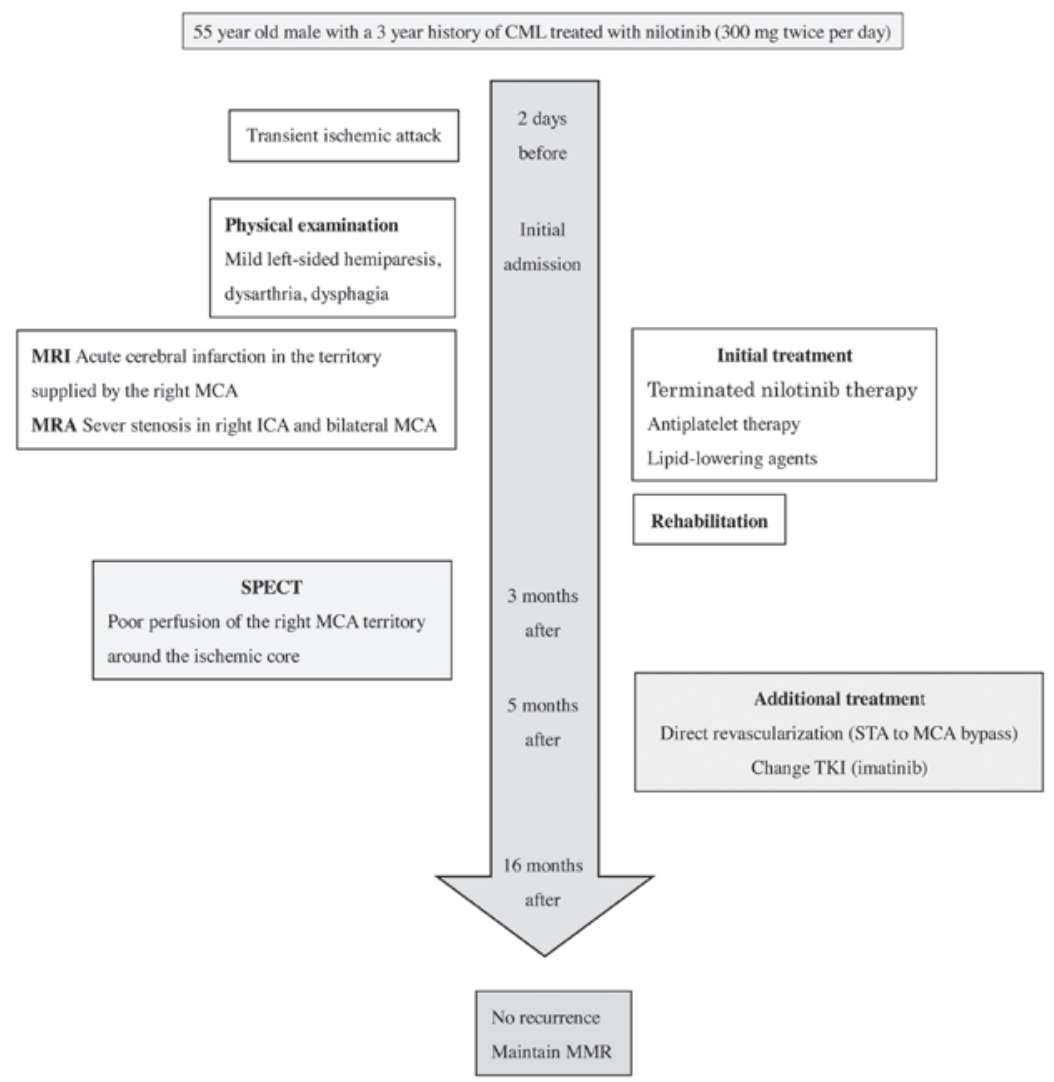

Figure 1. Timeline of the patient's clinical course. MRI, magnetic resonance imaging; MCA, middle cerebral artery; MRA, magnetic resonance angiography; ICA, internal carotid artery; SPECT, single-photon emission computed tomography; STA, superficial temporal artery; TKI, tyrosine kinase inhibitor; MMR, major molecular remission; CML, chronic myeloid leukemia.

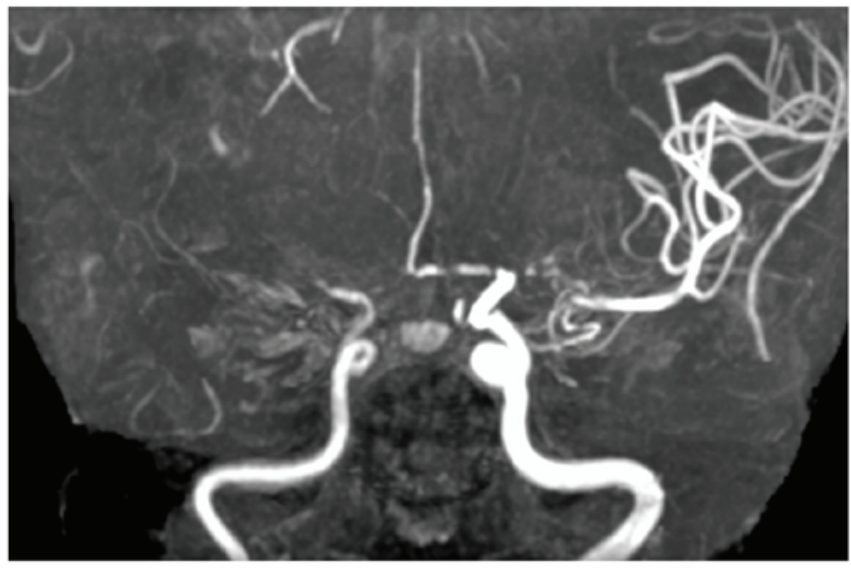

Figure 2. Time-of-flight magnetic resonance angiography (maximum intensity projection) showing severe narrowing and reduction of blood flow in the right internal carotid artery, occlusion of the right MCA and anterior cerebral artery, and severe narrowing of the left MCA. MCA, middle cerebral artery.

Occupational and Environmental Health Hospital (Kitakyushu, Japan). The neurological examination revealed mild left-sided hemiparesis, dysarthria and dysphagia. Magnetic resonance imaging (MRI) revealed acute cerebral infarction in the area supplied by the right middle cerebral artery (MCA). Magnetic resonance angiography (MRA) revealed severe stenosis in the right internal carotid artery (ICA) and left MCA (M1), without Moyamoya vessels (Fig. 2). Vessel wall magnetic resonance imaging (VW-MRI) revealed diffuse concentric thickening
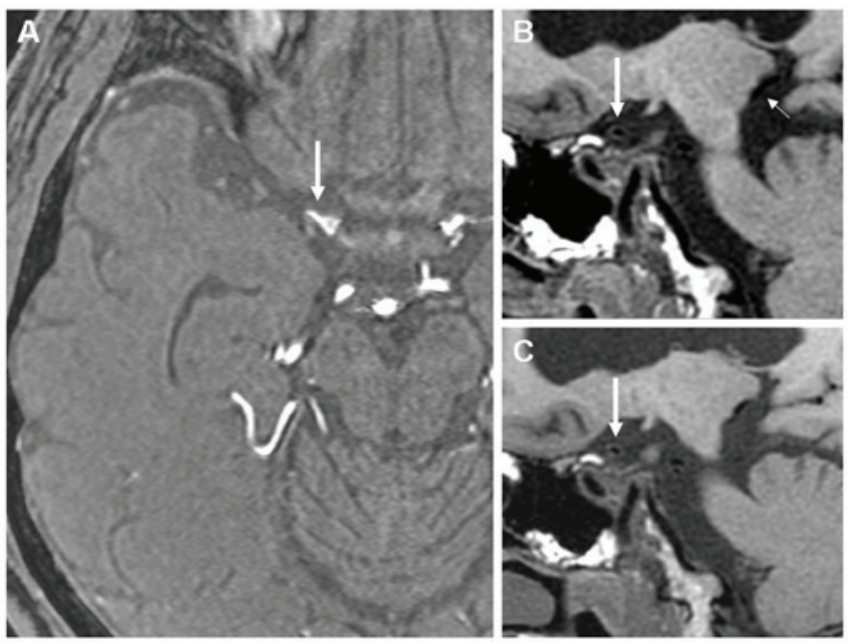

Figure 3. (A) Time-of-flight magnetic resonance angiography showing severe narrowing in the right MCA (arrow). (B) VW-MRI showing diffuse concentric thickening of the vessel wall in the MCA. The eccentricity index is calculated by using the following formula: (Maximal thickness - minimal thickness)/maximal thickness. This area is defined as a concentric plaque (big arrow). The remaining portion of the vessel exhibits no thickening (small arrow). (C) Follow-up VW-MRI 16 months later showing no notable thickening of the vessel wall (arrow). Note: The VW-MRI parameters consisted of a high-resolution fat-suppressed 3-dimensional fast spin echo proton density-weighted imaging (CUBE) sequence, which was acquired with the following parameters: Repetition time $1,000 \mathrm{msec}$, echo time $11.8 \mathrm{msec}$, NEX 1, echo-train length 32, band width $83.3 \mathrm{kHz}$, imaging time $4 \min 59 \mathrm{sec}$; a field of view of $24 \times 24 \times 17.2 \mathrm{~cm}^{3}$, and voxel size of $0.46 \times 0.46 \times 0.5 \mathrm{~mm}^{3}$. The images were acquired with the black blood technique and in the sagittal plane covering the whole brain. MCA, middle cerebral artery; VW-MRI, vessel wall magnetic resonance imaging. 


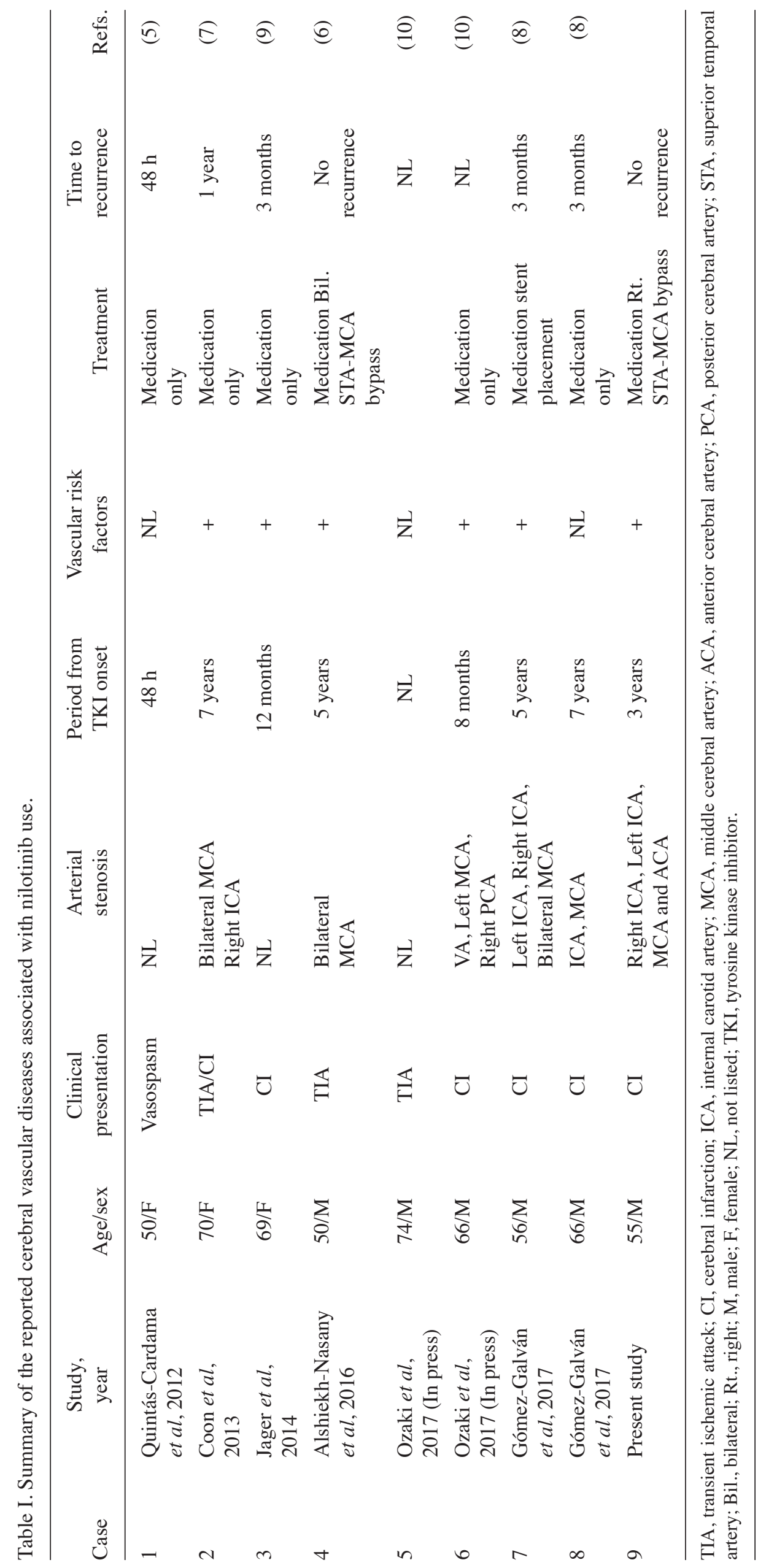


of the vessel wall of the right intracranial ICA and MCA, and the left MCA. Those findings were different from those of ordinary atherosclerosis, which is characterized by segmental and eccentric wall thickening (Fig. 3A and B). The results of the blood tests demonstrated a mildly elevated low-density lipoprotein (LDL) cholesterol level (139 mg/dl). The results of the serological examination for infectious, collagen and hormonal diseases were unremarkable. The patient had no history of hypertension, arrhythmia, or diabetes mellitus; he occasionally consumed alcohol and had a sporadic smoking habit; he had no family history of stroke or vascular diseases. Thus, the clinical diagnosis was cerebral infarction associated with nilotinib use.

Nilotinib maintenance therapy was terminated and the patient was treated with antiplatelet therapy and a high-potency statin. After 3 months of rehabilitation, his symptoms gradually improved.

An additional examination for brain ischemia was then performed. Cerebral angiography revealed no notable atherosclerosis in the cervical carotid artery and aortic arch, apart from the intracranial lesions previously detected by MRA. Single photon-emission computed tomography imaging revealed poor perfusion of the area supplied by the right MCA around the ischemic core. Subsequently, the patient underwent direct revascularization [superficial temporal artery (STA) to MCA bypass] 5 months after the initial admission.

Thereafter, the patient developed no recurrence of brain ischemia. Follow-up MRI examination 16 months after the initial presentation also revealed no marked changes of the cerebral arteries (Fig. 3C), and the patient was able to resume his daily activities. Nilotinib was replaced with imatinib after the initial admission, and major molecular remission has been maintained as at August 2018.

\section{Discussion}

Although PAOD is a well-known nilotinib-associated VAE, nilotinib-associated CVD is extremely rare. To the best of our knowledge, only 8 such cases have been published in the literature to date (Table I). Nilotinib-associated CVD is relatively common in middle-aged men, with development of brain ischemia due to severe stenosis or occlusion in the main trunk of the cerebral arteries. In most cases, the onset of symptoms occurs several months or years after the initiation of nilotinib treatment, except in one case. In addition, almost all the patients had some additional vascular risk factors, which were not all attributable to nilotinib use. Our patient was a middle-aged man with a smoking habit and a mildly elevated LDL cholesterol level, who developed cerebral infarction due to severe stenosis of the major cerebral arteries 3 years after nilotinib treatment initiation, similar to previous cases.

The pathogenesis of nilotinib-associated CVD remains unclear. Previous studies have demonstrated that the discoidin domain receptor 1 (DDR1), platelet-derived growth factor receptor (PDGF-R) and C-kit may be associated with systemic VAEs during nilotinib use as off-target activity (1). Among those, DDR1 plays a role in atherosclerosis, and nilotinib has a particularly high affinity for this receptor, compared with that for BCR/ABL1 (4). PDGF-R and C-kit may also potentially provide explanations for the vascular toxicity, since they are both involved in the regulation of vascular and perivascular cells $(1,4)$. Furthermore, previous studies have suggested that nilotinib exerts direct pro-atherogenic and anti-angiogenic effects on endothelial cells (11). An altered endothelial cell function with increased expression of cytoadhesive cell surface molecules and consecutive pro-atherogenic changes in the vessel wall are considered to be a key mechanism underlying the VAE-promoting action of nilotinib (12). In addition, nilotinib exerts metabolic effects, including an increase in cholesterol and fasting glucose levels (1). Thus, previous studies have suggested that nilotinib induces systemic arterial stenosis by its direct pro-atherogenic effects, and inhibits recanalization and reperfusion via its anti-angiogenic effects, thus leading to systemic VAEs.

VW-MRI can provide information on vessel wall changes to distinguish among different CVDs. In atherosclerosis, the atherosclerotic plaques tend to appear as asymmetrical vascular wall thickenings (eccentricity) relative to other vascular pathologies. Conversely, symmetrically thickened walls are usually observed in vasculitis, Moyamoya disease, and reversible cerebral vasoconstriction syndrome (13). In the present case, VW-MRI revealed diffuse concentric thickening of the vessel wall of the right intracranial ICA and MCA, and the left MCA. Additionally, the eccentricity index [calculated as (maximal thickness - minimal thickness)/maximal thickness] was 0.1-0.2; if the index exceeds 0.5, the lesion is defined as an eccentric plaque (13). The findings of follow-up MRI did not match the future development of Moyamoya disease, which is characterized by progressiveness. These findings in the present case indicated that the morphological changes of the vessel wall were inconsistent with those of ordinary simple atherosclerotic disease. We could not perform pathological examination of the intracranial arteries, which exhibited severe stenosis, due to the clinical and surgical limitations. Instead, the pathological study of the STA, which was retrieved during surgery, revealed no notable atherosclerotic and inflammatory changes or wall thickening. Nilotinib induces vasculitis and/or severe atherosclerotic changes in the entire circumference of the main trunk of the cerebral arteries under certain conditions, such as smoking, diabetes mellitus, hypertension and hypercholesterolemia. The findings on VW-MRI in the present case suggested that those morphological changes were potentially caused by ordinary atherosclerosis and vasculitis, as CVDs associated with nilotinib use were limited to the intracranial arteries.

Patients who develop nilotinib-associated CVD are generally managed by anticoagulant or antiplatelet therapy, elimination of risk factors, a reduced TKI dose and conversion to another TKI (5-9). Yet, the majority of the patients experienced recurrent CVD within 1 year after their initial symptoms. Only 2 patients, including our patient, underwent STA-MCA bypass surgery with medical treatment and experienced no recurrence of CVD. The number of similar cases and follow-up period are limited; however, in the present case, the severe stenosis in the MCA and ICA was not progressed and the patient's neurological status was not changed, as indicated by follow-up MRI. Thus, surgical revascularization may improve prognosis in patients with nilotinib-associated CVD, which manifests with any critical and symptomatic intracranial arterial stenosis.

In conclusion, nilotinib-associated CVD is rare, but its incidence may increase as the use of nilotinib increases among patients with CML. Thus, clinicians should be aware of the 
risk factors of nilotinib-associated CVD and perform careful long-term follow-up of patients for several years. As regards the management of nilotinib-associated CDV, surgical revascularization may improve the prognosis of such patients.

\section{Acknowledgements}

Not applicable.

\section{Funding}

No funding was received.

\section{Availability of data and materials}

Not applicable.

\section{Authors' contributions}

KS drafted the manuscript. JY, TS and YN revised the content and advised constructive feedbacks. KS, JY, RM and TK performed this surgery. SK and ST analysed all images and drafted parts of the manuscript. SN wrote the final draft. All the authors have read and approved the final version of this manuscript.

\section{Ethics approval and consent to participate}

Not applicable.

\section{Consent for publication}

Informed consent was obtained from the patient about the publication of the case details and any associated images.

\section{Competing interests}

The authors declare that they have no competing interests.

\section{References}

1. Pasvolsky O, Leader A, Iakobishvili Z, Wasserstrum Y, Kornowski R and Raanani P: Tyrosine kinase inhibitor associated vascular toxicity in chronic myeloid leukemia. Cardiooncology 1:5, 2015.

2. Chai-Adisaksopha C, Lam W and Hillis C: Major arterial events in patients with chronic myeloid leukemia treated with tyrosine kinase inhibitors: A meta-analysis. Leuk Lymphoma 57: 1300-1310, 2016.

3. Saglio G, Kim DW, Issaragrisil S, le Coutre P, Etienne G, Lobo C, Pasquini R, Clark RE, Hochhaus A, Hughes TP, et al; ENESTnd Investigators: Nilotinib versus imatinib for newly diagnosed chronic myeloid leukemia. N Engl J Med 362: 2251-2259, 2010.

4. Aichberger KJ, Herndlhofer S, Schernthaner GH, Schillinger M, Mitterbauer-Hohendanner G, Sillaber C and Valent P: Progressive peripheral arterial occlusive disease and other vascular events during nilotinib therapy in CML. Am J Hematol 86: 533-539, 2011.

5. Quintás-Cardama A, Kantarjian H and Cortes J: Nilotinibassociated vascular events. Clin Lymphoma Myeloma Leuk 12: 337-340, 2012

6. Alshiekh-Nasany R, Zidan A and Martinez C: Extensive intracranial arterial stenoses in conjunction with the use of tyrosine kinase inhibitor Nilotinib. Clin Case Rep 4: 1184-1186, 2016.

7. Coon EA, Zalewski NL, Hoffman EM, Tefferi A and Flemming KD: Nilotinib treatment-associated cerebrovascular disease and stroke. Am J Hematol 88: 534-535, 2013.

8. Gómez-Galván JB, Borrego S, Tovar N and Llull L: Nilotinib as a risk factor for ischaemic stroke: A series of three cases. Neurologia 32: 411-413, 2017 (In Spanish).

9. Jager NG,Stuurman FE,Baars JW and Opdam FL: Cerebrovascular events during nilotinib treatment. Neth J Med 72: 113-114, 2014.

10. Ozaki T, Nakamura H, Izutsu N, Masaie H, Ishikawa J and Kinoshita M: Intracranial stenting for nilotinib treatment-associated cerebrovascular stenosis in chronic myeloid leukemia. Interv Neuroradiol 23: 527-530, 2017.

11. Hadzijusufovic E, Albrecht-Schgoer K, Huber K, Hoermann G, Grebien F, Eisenwort G, Schgoer W, Herndlhofer S, Kaun C, Theurl M, Sperr WR, et al: Nilotinib exerts direct pro-atherogenic and anti-angiogenic effects on vascular endothelial cells: A potential explanation for drug-induced vasculopathy in CML. Blood 122: 257, 2013.

12. Hadzijusufovic E, Albrecht-Schgoer K, Huber K, Hoermann G, Grebien F, Eisenwort G, Schgoer W, Herndlhofer S, Kaun C, Theurl M, et al: Nilotinib-induced vasculopathy: Identification of vascular endothelial cells as a primary target site. Leukemia 31: 2388-2397, 2017.

13. Choi YJ, Jung SC and Lee DH: Vessel wall imaging of the intracranial and cervical carotid arteries. J Stroke 17: 238-255, 2015. 